\title{
Aplicación de Ondas Sónicas y Ultrasónicas como Medio de Control del Ataque de Termitas Subterráneas Reticulitermes flavipes (Kollar) en Madera de Pino Radiata.
}

\section{Application of Sonic and Ultrasonic Waves as Means of Control of Underground Termites Reticulitermes flavipes (Kollar) in Radiata Pine wood.}

\section{J.T. KARSULOVIC ${ }^{1}$, A. BOZO ${ }^{1}$, J. ARAYA ${ }^{2}$, Y. VARGAS ${ }^{3}$, V. GAETE ${ }^{1}$, B. TEJER ${ }^{4}$}

1 Académicos Departamento de Ingeniería de la Madera, Facultad de Ciencias Forestales, Universidad de Chile. Santiago.Chile.

e-mail: tkarsulo@uchile.cl

2 Académico Departamento de Sanidad Vegetal, Facultad de Ciencias Agronómicas, Universidad de Chile. Santiago.Chile.

3 Académico Departamento de Física, Facultad de Ingeniería, Universidad de Santiago de Chile. Santiago.Chile.

${ }^{4}$ Memorante Departamento de Ingeniería de la Madera, Facultad de Ciencias Forestales, Universidad de Chile. Santiago.Chile.

Autor para correspondencia: tkarsulo@uchile.cl

Recibido: 26.01.2006. Aceptado: 28.04.2008.

\section{RESUMEN}

Se analizó el comportamiento de las termitas al aplicar campos sónicos y ultrasónicos con diferentes frecuencias, niveles de energía y formas de onda, de modo de establecer las condiciones para crear una barrera física frente a la acción de las termitas en probetas de madera de pino radiata.

Los ensayos a nivel del rango ultrasónico se efectuaron con frecuencias de 100,500 y $1000 \mathrm{kHz}$ en probetas de 1x4x10 cm mediante una adaptación de la norma Europea EN118.

La aplicación de irradiación a nivel sónico se realizó sobre probetas de diferentes escuadrías comerciales en dirección transversal considerando distintas orientaciones de los anillos de crecimiento (radial, tangencial y mixto) y tres niveles de densidad (baja, media y alta). Para este efecto se diseñó un sistema constituido de un mini-termitero conteniendo 500 termitas unido a la muestra y una fuente de alimentación de modo de establecer un flujo continuo de termitas a través de una perforación central practicada en la muestra.

En el rango ultrasónico se obtuvo que con una frecuencia de $500 \mathrm{kHz}$, fue posible inhibir la alimentación de las termitas mientras el campo acústico se mantiene activo.

En el rango audible estudiado, $(16 \mathrm{~Hz}$ a $20 \mathrm{kHz})$ se observó que a una frecuencia de $80 \mathrm{~Hz}$ se inhibe el paso de las termitas a través de la muestra alterándose drásticamente su comportamiento; en tiempos superiores a una hora de irradiación continúa, la rapidez de recuperación es parcial, disminuyendo las tasas de alimentación y la sobrevivencia, lo que evidencia la generación de un efecto fisiológico irreversible.

Palabras claves: Ondas acústicas, control, termitas subterráneas, pino radiata. 


\section{SUMMARY}

The behavior of the termites was analyzed when applying sonic and ultrasonic waves with different frequencies, levels of energy, and waveform to establish the conditions to create a physical barrier to the action of the termites in wood specimens of radiata pine.

The tests at ultrasonic level were conducted using frequencies of 100,500 and $1000 \mathrm{kHz}$ in specimens of $1 \times 4 \times 10 \mathrm{~cm}$ by means of an adaptation of European Standard EN118.

The application of irradiation at sonic level was made on specimens with different cross section in transverse direction considering different orientation of growth rings (radial, tangential and mixed) and three levels of density (low, medium and high). For this effect was constituted a system of a scale termite nest containing 500 termites with the wood specimen and a feed supply to establish a continuous flow of termites through a practiced central perforation in the specimen.

In the ultrasonic rank it was obtained that with a frequency of $500 \mathrm{kHz}$ it is possible to inhibit the feeding of the termites while the acoustic field stays assets.

In the studied audible rank, $(16 \mathrm{~Hz}$ to $20 \mathrm{kHz})$ it was observed that to a frequency of $80 \mathrm{~Hz}$ the passage of the termites through the sample was inhibited and they altered their behavior; in times superior to one hour of continuous irradiation the rapidity of recovery is partial, diminishing the rates of feeding and survival with generation of irreversible a physiological effect.

Key words: Waves, termites, radiata pine.

\section{INTRODUCCIÓN}

Las termitas juegan un importante rol ecológico en la naturaleza, sin embargo, siendo la celulosa su principal fuente de alimentación éstas constituyen un grave agente de deterioro para la madera en servicio.

Hasta hace algunos años en Chile existían solo dos familias de termitas, Kalotermidae y Termopsidae, agregándose con la introducción de la termita subterránea la familia Rhinotermitidae a la cual pertenece la especie. Reticulitermes hesperus Banks, según los primeros reportes, sería originaria de Estados Unidos e introducida en Chile en la década de los setenta (Camousseight, 1998), sin embargo, actualmente se afirma que se trataría de la especie Reticulitermes flavipes.

Las especies del género Reticulitermes son la mayor plaga que se encuentra infestando estructuras en numerosos países del mundo. Se estima que solo en Estados Unidos las termitas causan sobre 3 billones de dólares anuales en daños a la madera estructural, de este monto el $80 \%$ es atribuible a la termita subterránea (Lewis, 1997).

En Chile, se ha detectado la presencia de la termita subterránea en la Región Metropolitana y Quinta Región. En Santiago, en una prospección realizada entre los años 1997 y 1998, se detectaron 80 focos de infestación en 29 comunas, (INTEC-INFOR, 1997) no cabiendo dudas que se ha producido un significativo incremento de su expansión en estos últimos años dado al aumento de las denuncias en casi todas las comunas de Santiago. En la quinta Región, durante los tres años en que fueron estudiados focos de la térmica subterránea, desde 2002 a 2004, éstos aumentaron de 30 a 56 focos de diferentes magnitud (INIA, 2004). A pesar de esto, lamentablemente no se han establecido políticas gubernamentales que tiendan a tratar de disminuir el impacto de la plaga que está afectando especialmente a los niveles sociales de menores recursos.

El hábito subterráneo de la termita, hace difícil su detección y les permite expandirse a través de amplias extensiones bajo el suelo, además ellas pueden acceder en la superficie a piezas de madera alejadas del suelo a través de la construcción de túneles sobre paredes de cualquier material de que esté constituida su edificación.

Las termitas son insectos relativamente pequeños (entre 3 y $10 \mathrm{~mm}$ ) y viven en asociaciones constituidas de diferentes castas, siendo los juveniles desde la segunda muda en adelante, a excepción de los soldados, 
los que realizan las galerías en la madera que atacan. Son especies longevas de complejo ciclo biológico y singulares relaciones entre individuos, tales como: secreción de feromonas de alarma y de agregación, alimentación trofaláctica, canibalismo, necrofagia, oofagia y emisión de ruidos.

Las termitas requieren de condiciones ambientales específicas para sobrevivir, la humedad y la temperatura son las variables que más las afectan (Smith \& Rust, 1993 a / b y 1994). Para avanzar en áreas expuestas en búsqueda de alimento construyen túneles de comunicación, fabricados con una mezcla de saliva, heces y material de sustrato, que les proveen un ambiente húmedo y las protegen parcialmente de enemigos naturales. Por otra parte, las termitas evitan al máximo las variaciones de temperatura y presentan una respuesta negativa a la luz. Grace et al. (1995) estudiando el comportamiento de Reticulitermes flavipes y Reticulitermes santonensis establece que las termitas son capaces de orientarse por si mismas a lo largo de los senderos utilizando mecanismos quimiotáctiles y quimiocinéticos.

La erradicación total de las termitas no es posible y los esfuerzos deben orientarse a controlar la existencia de la población de insectos y limitar o disminuir los riesgos a las construcciones, a través de la implementación de estrategias integradas de manejo de la plaga (Morris, 2000). Para un adecuado manejo de las termitas es necesario disponer de métodos de prevención y control. Para la prevención se utilizan diferentes técnicas, consistentes en el establecimiento de barreras físicas y químicas. En general, el uso de barreras físicas esta concebido para tratamientos de pre-construcción de una edificación (uso de arenas, mallas de acero, plásticos impregnados, etc.). Las barreras químicas se utilizan tanto como tratamientos preventivos como curativos en pre y post construcción y tienen por objetivo la exclusión de las termitas subterráneas de las estructuras. Los métodos de control curativos consisten en la aplicación de tratamientos químicos y no químicos, como por ejemplo, se han utilizado el dióxido de carbono (Delate et al. 1995) aplicable en espacios cerrados, utilización de aire caliente (Woodrow y Grace, 1998), nitrógeno líquido (Lewis, 1997), utilización de microondas y aplicación de electricidad de alto voltaje (Mashek, 1998). Los tratamientos químicos en áreas localizadas consisten en la perforación y posterior inyección de insecticidas en las galerías construidas por las termitas a través de pequeñas perforaciones.

Para la aplicación de métodos curativos es necesario disponer de sistemas de inspección o detección de la actividad de las termitas en elementos en servicio. Para éste objetivo se han desarrollado diferentes técnicas, tales como la emisión acústica (Lemaster et al, 1997, Yanase et al. 1998; Mankin et al. 2002) ondas de esfuerzo (Ross et al. 1997; De Groot et al. 1998), ultrasonido (Wilcox, 1988), detección de gases que emiten las termitas durante su metabolismo (Metano y dióxido de carbono) imágenes infrarrojas, microondas, rayos $\mathrm{x}$, etc. (INIA, 2004)

Este trabajo pretende efectuar un nuevo aporte a las técnicas ya existentes para el control de termitas, teniendo por objetivo estudiar la aplicación de ondas sónicas y ultrasónicas como barreras físicas, no existiendo trabajos reportados al respecto. Se plantea la hipótesis que las ondas ultrasónicas producen efectos demostrados sobre los sistemas biológicos de los animales. En base a esto se postula que las termitas pueden ser susceptibles a su acción, a una determinada frecuencia e intensidad, pudiendo producir alteraciones en sus mecanismos de comunicación, alimentación u otros cambios biofísicos y/o bioquímicos que ocasionen efectos inmediatos o mediatos en su comportamiento o sobrevivencia. Respecto a las ondas sónicas se postula que las vibraciones mecánicas inducidas en piezas de madera a frecuencia de resonancia del insecto, así como la excitación con frecuencias correspondientes a los sonidos que emiten los soldados en sus señales de alarma y por otra parte las que se generan como emisión acústica durante la alimentación de las termitas podrían alterar el comportamiento de las termitas, inhibiendo el ataque a la madera.

El ultrasonido comprende ondas elásticas con frecuencias de vibración de partículas sobre el rango del umbral de la audición humana, es decir, sobre $20 \mathrm{kHz}$. La propagación de las vibraciones ultrasónicas en los distintos medios es muy análoga a la propagación del sonido, si bien su absorción o grado de atenuación es mucho mayor. El interés práctico de la utilización del ultrasonido reside ya sea en el aprovechamiento de la energía emitida (Dispersiones, reacciones químicas, generación de calor, etc.) o 
bien en el análisis del rayo una vez que se le hace pasar por un medio (inspección física de materiales, estudios de absorción, etc.) El haz ultrasónico tiene diversas acciones y comportamientos que dependen de la energía, la longitud de onda y las interfaces que se interponen en su trayectoria. Cuando un haz ultrasónico es emitido a través de un medio heterogéneo tal como la madera o tejidos blandos su intensidad se reduce o atenúa a través de varios mecanismos que incluyen scattering, absorción, reflexión, difracción y refracción. La absorción del ultrasonido ocurre cuando la energía vibracional de la onda es disipada en su movimiento molecular interno y éste último en calor (Kenneth, 1988).

Los ultrasonidos pueden modificar de alguna manera la materia mediante dos mecanismos, el térmico y la cavitación. En el primer caso la absorción de la energía ultrasónica genera calor, en potencias bajas el efecto es despreciable como en las aplicaciones de diagnósticos médicos (ultrasonografía). En el segundo caso, la cavitación se refiere a la formación y colapso de cavidades en un líquido en las que o bien existe un alto vacío o se encuentran saturada de vapor de dicho líquido o con los gases disueltos. En una cavitación la continuidad del medio es destruida, este fenómeno puede observarse como un efecto no lineal (Shutilov, 1988). Extensos trabajos se han realizado sobre la acción del ultrasonido sobre sistemas químicos y en moléculas de interés biológico (Kenneth, 1988).

Todos los insectos contienen microscópicos cuerpos gaseosos estables, los cuales pueden oscilar bajo la influencia del ultrasonido, generando flujos en los tejidos adyacentes que pueden ser responsables de los efectos que se han observado en muchos estudios con huevos, larvas, y estados prepupales (Kenneth, 1988 ).

Es de interés en éste trabajo hacer notar que las termitas están compuestas de alrededor de una 70\% de líquido de carácter acuoso. El líquido que circula por la cavidad del cuerpo de las termitas (hemolinfa) consta de una parte líquida o plasma y un conjunto de células libres en suspensión, llamadas corpúsculos sanguíneos o hemócitos. Si bien los líquidos polares tienen una absorción baja a moderada es de interés considerar que en el proceso metabólico de las termitas se libera ácido acético, el cual es absorbido y oxidado por el termes para cubrir sus necesidades de energía, la absorción del ácido acético alimenta al termes y actúa en beneficio de los mismos protozoarios (las termitas se alimentan de la celulosa mediante endosimbiosis) ya que impide la acumulación de éste ácido que le es dañina (Hungate, 1975). Se ha observado que la máxima absorción del ultrasonido en el ácido acético se produce a una frecuencia de $500 \mathrm{kHz}$ lo que hace que sea de interés la irradiación de las termitas a ésta frecuencia.

La radiación ultrasónica ejerce una función catalítica sobre reacciones químicas y es capaz de provocar ciertos efectos característicos, especialmente electroquímicos. Se supone que la verdadera causa de su acción sobre las reacciones químicas se debe a la cavitación. Entre algunos de los efectos se encuentra la activación de la polimerización, efecto oxidantes en disoluciones acuosas por la tendencia a la formación de radicales libres que luego producen diversas reacciones secundarias, tales como la inversión de sacarosas, hidrólisis de esteres, oxidación, etc. (Kenneth, 1988).

Se ha demostrado que algunos insectos y otros animales inferiores mueren en poco tiempo por efecto del ultrasonido y los organismos embrionarios presentan desenvolvimiento anormal. Si los órganos sobre los que se quiere actuar están sumergidos los efectos son más violentos, debido principalmente al aumento de la superficie expuesta. La cavitación produce la destrucción de la membrana de las células dependiendo éste hecho de la frecuenta de radiación, entre otros factores. En general, todas las variables físicas que inciden en la cavitación influyen secundariamente en el efecto producido sobre los organismos (Almagro, 1967). La respuesta de los adultos y larvas de insectos al ultrasonido está significativamente influenciada por la presencia de microscópicos poros de aire (Child et. al. 1989).

Este trabajo estudió el efecto de la cavitación sobre las termitas con el objetivo de poder establecer una metodología para utilizar este fenómeno como un medio de control de la madera contaminada residual, producto del reemplazo de elementos o material de demolición de construcciones atacadas. Este método podría ser utilizado como alternativa menos contaminante que la quema del material infestado y el depósito de la madera en vertederos. 


\section{MATERIALES Y MÉTODOS}

Recolección y Mantención de Termitas: Las termitas utilizadas en los ensayos provinieron de dos colonias ubicadas en las comunas de San Bernardo y Santiago (R. Metropolitana). La captura se efectuó mediante cebos diseñados y construidos para este efecto, utilizando tubos de PVC de $75 \mathrm{~mm}$ de diámetro y $250 \mathrm{~mm}$ de largo, rellenos con cartón corrugado como fuente de alimentación; a los tubos se les practicó ranuras de $5 \mathrm{~mm}$ de ancho a través de su periferia para permitir la entrada de las termitas. Los cebos fueron enterrados en lugares adecuados durante un período de 20 días durante los meses de Septiembre, Octubre y Noviembre del año 2004, durante los meses de verano no fue posible la captura debido posiblemente a las altas temperaturas reinantes.

Con las termitas capturadas se establecieron cinco colonias las que fueron instaladas en una sala climatizada con ambiente controlado de temperatura $\left(25^{\circ} \mathrm{C}\right.$ a $\left.27^{\circ} \mathrm{C}\right)$ y humedad relativa $(70 \%$ a $80 \%)$ de modo de otorgarles las condiciones óptimas para su mantención y desarrollo. La constitución de los termiteros se realizó mediante receptáculos de vidrio de $60 \mathrm{~cm}$ de longitud por $45 \mathrm{~cm}$ de ancho y $30 \mathrm{~cm}$ de alto con tapa y orificio de aireación, cubierto con rejilla metálica. En su interior se introdujo un sustrato formado de gravilla mezcla de tierra y arena de cuarzo y material inductor (pequeños trozos de madera de pino radiata); para mantener la humedad del sustrato se le adicionó diariamente una cantidad de agua destilada. Como fuente de alimentación se instalaron dentro de los termiteros paquetes de seis tablillas de madera de pino radiata de $5 \mathrm{~mm}$ de espesor, $60 \mathrm{~mm}$ de ancho y $200 \mathrm{~mm}$ largo, unidas entre ellos con amarras de alambre; de acuerdo a los requerimientos de los ensayos se fueron extrayendo los paquetes y coleccionando las termitas en la cantidad necesaria, para luego retornar los paquetes al termitero.

Ensayos Ultrasónicos: Para estos ensayos se utilizó la norma Europea EN 118 con algunas modificaciones, en efecto, en lugar de una película protectora como específica la norma se aplicó un campo ultrasónico como barrera, empleándose frecuencia de irradiación ultrasónica de 100, 500 y 1000kHz. La variable respuesta a analizar correspondió a la eliminación o inhibición a la alimentación de las termitas por efecto del campo ultrasónico. La generación del campo ultrasónico se realizó mediante un generador de pulso ultrasónico Panametrics Ultrasonic, pulser/receiver, modelo 5058PR y transductores piezoeléctricos de contacto Panametrics de onda longitudinal, modelo Videoscan P/N V194-RB, la intensidad máxima irradiada por estos transductores es de $0,024 \mathrm{~W} / \mathrm{cm}^{2}$.

Se utilizaron 12 probetas de pino radiata, tres de ellas utilizadas como testigos, de una densidad promedio de $613 \mathrm{~kg} / \mathrm{m}^{3}$ a $12 \%$ de contenido de humedad, con una sección transversal de 10x40mm y $100 \mathrm{~mm}$ de longitud, selladas en sus cantos con pinturas de aluminio para evitar pérdidas de humedad. Según las especificaciones de la norma EN 118, modificadas de acuerdo a los objetivos del estudio, se procedió a fijar tubos de vidrio de $25 \mathrm{~mm}$ de diámetro y $110 \mathrm{~mm}$ de longitud abiertos en ambos extremos; a las probetas mediante un adhesivo epóxico. En los tubos se introdujo arena de cuarzo hasta una altura de $70 \mathrm{~mm}$ desde la base del tubo. La arena fue permanentemente humedecida con agua destilada en proporción de un volumen de agua por cuatro de arena; a una distancia de $1 \mathrm{~cm}$ de la base se agrego $0,2 \mathrm{~g}$ de madera de cría, la que sirvió de alimento para las termitas durante el período de adaptación, previo a la realización de los ensayos. Luego se introdujeron en los 12 tubos de ensayo 250 termitas obreras. El peso del conjunto se mantuvo constante durante todo el período de ensayo, agregándose agua destilada diariamente para mantener la humedad inicial. El set de ensayo se instaló en una cámara de clima diseñada especialmente de modo que las irradiaciones se efectuaran en condiciones controladas de temperatura y humedad relativa (Fig. 1). 


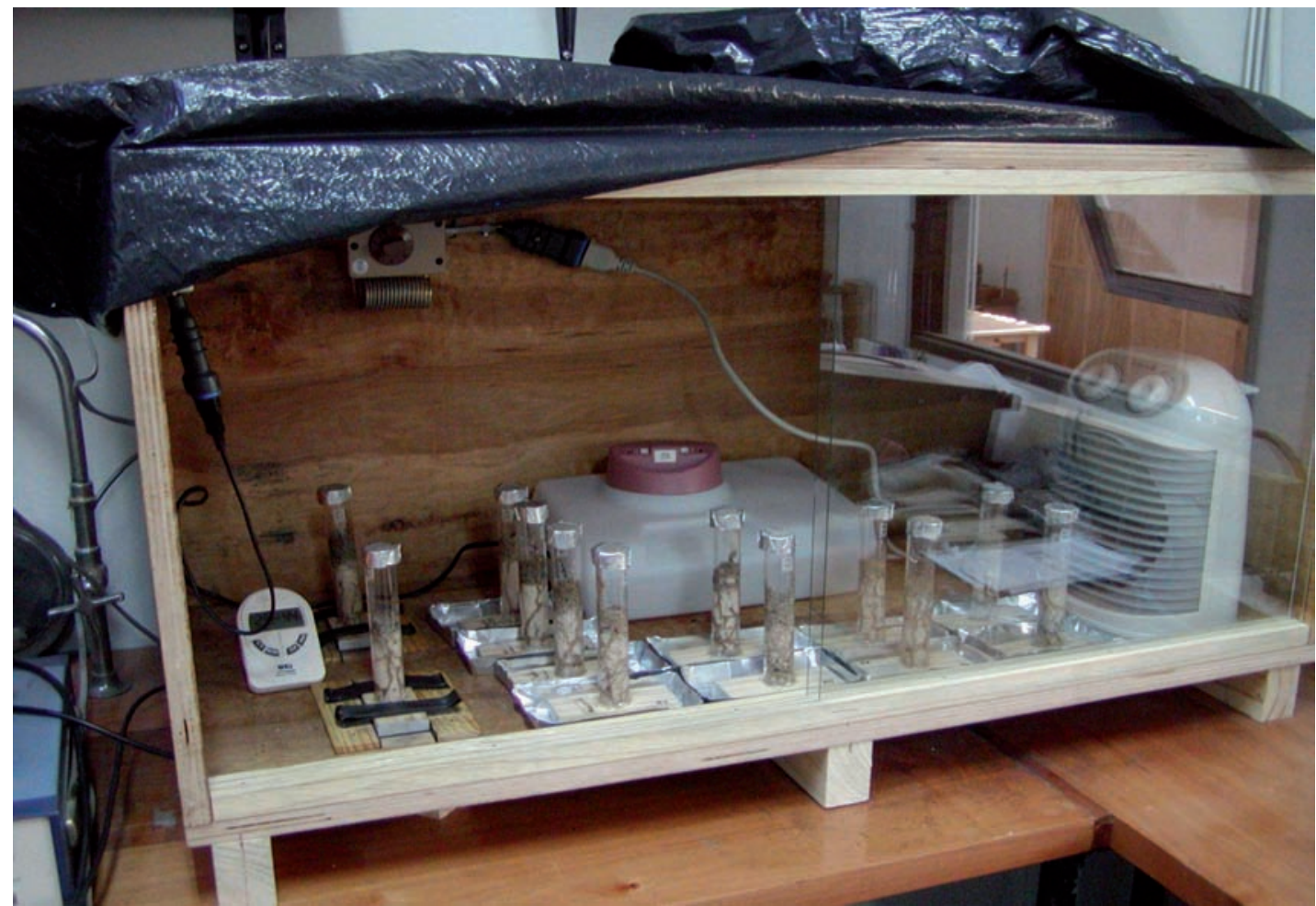

Figura 1. Cámara de clima utilizada en la primera etapa del estudio.

Después de siete días en que las termitas se adecuaron a sus nuevas condiciones las probetas fueron irradiadas durante dos horas diarias con las tres frecuencias establecidas, completando 20 horas de irradiación en tiempo acumulado. La evaluación de los resultados se efectuó mediante un análisis cualitativo, según lo establecido por la Norma Europea EN 118 que se presenta en la Tabla 1.

TABLA 1. Clasificación del ataque según intensidad del daño.

\begin{tabular}{|c|l|}
\hline $\begin{array}{c}\text { Grado de } \\
\text { ataque }\end{array}$ & \multicolumn{1}{|c|}{ Descripción } \\
\hline 0 & Ningún ataque \\
\hline 1 & Tentativa de ataque: arañazos o roeduras superficiales cuya profundidad no se puede medir. \\
\hline 2 & $\begin{array}{l}\text { Ataque ligero: ataque superficial (menos de } 1 \mathrm{~mm} \text { ) y limitado en extensión a } 1 / 4 \text { de la superficie } \\
\text { expuesta como máximo, o una perforación única de profundidad inferior a } 3 \mathrm{~mm}, \text { sin que exista otra } \\
\text { tasa de ataque. }\end{array}$ \\
\hline 3 & $\begin{array}{l}\text { Ataque medio: ataque superficial (menos de } 1 \mathrm{~mm} \text { ) que se extiende a más de } 1 / 4 \text { de la superficie } \\
\text { expuesta o erosión (de } 1 \mathrm{a} 3 \mathrm{~mm} \text { ) sobre una superficie inferior o igual a } 1 / 4 \text { de la expuesta } 0 \\
\text { perforaciones puntuales superiores a } 3 \mathrm{~mm} \text {, pero que no se extiendan en cavernas o no atraviesen. }\end{array}$ \\
\hline 4 & $\begin{array}{l}\text { Ataque fuerte: erosión sobre más de } 1 / 4 \text { de la superficie expuesta o ataque penetrante superior a } \\
3 \mathrm{~mm} \text { extendiéndose en cavernas en el interior de la probeta o sin extenderse en cavernas, pero } \\
\text { atravesándola. }\end{array}$ \\
\hline
\end{tabular}

Fuente: Norma Europea EN 118, 1992. 
Irradiación de Termitas en Agua: Fueron sometidas a irradiación 270 termitas obreras sumergidas en agua destilada a las que se le aplicaron dos tratamientos de frecuencia de 11 y $20 \mathrm{kHz}$, con tres potencias, $5 \mathrm{~W}, 10 \mathrm{~W}$ y $15 \mathrm{~W}$, y a su vez cada una en tres tiempos $2 \mathrm{~min} ., 5 \mathrm{~min}$. y $10 \mathrm{~min}$. Para cada tratamiento se efectuaron tres repeticiones, tratándose en cada uno de ellas 10 termitas, tomando igual cantidad como testigo. Los ensayos se realizaron en vaso de precipitado, utilizando transductores de potencia tipo bocina escalonada y un generador de potencia de 500W, modelo LC 9301.

La variable respuesta fue el grado de mortalidad de las termitas bajo la acción directa de la irradiación. Los resultados obtenidos se evaluaron mediante un análisis de varianza utilizando un diseño trifactorial de efectos fijos, con tres repeticiones. Los factores analizados fueron la frecuencia, potencia y tiempo. Determinadas las diferencias de medias se realizó una prueba de intervalos de Duncan para establecer el grado de significancia de los factores.

Ensayos Sónicos: Con el objetivo de evaluar el efecto de las ondas acústicas como barrera al paso de las termitas, se desarrolló un set experimental que permitiese la aplicación de un campo sónico directamente a la madera con transito de termitas en su interior. Para eso se construyó un mini termitero constituido de dos receptáculos de PVC de $75 \mathrm{~mm}$ de diámetro y $150 \mathrm{~mm}$ de altura, donde uno de ellos cumplió función de nido y el otro como contenedor de fuente de alimentación. Entre ambos receptáculos se instalaron las probetas de ensayo, la que en su parte central se les practicó una perforación de $3 \mathrm{~mm}$, de modo de simular una galería. Los tres elementos fueron unidos mediante tubos de vidrio de nueve milímetros de diámetro (Fig. 2). En el receptáculo correspondiente al nido se introdujeron 500 termitas y en el de la fuente de alimentación se instalaron sobre el sustrato, construido de arena de cuarzo, trozos de madera de pino radiata.

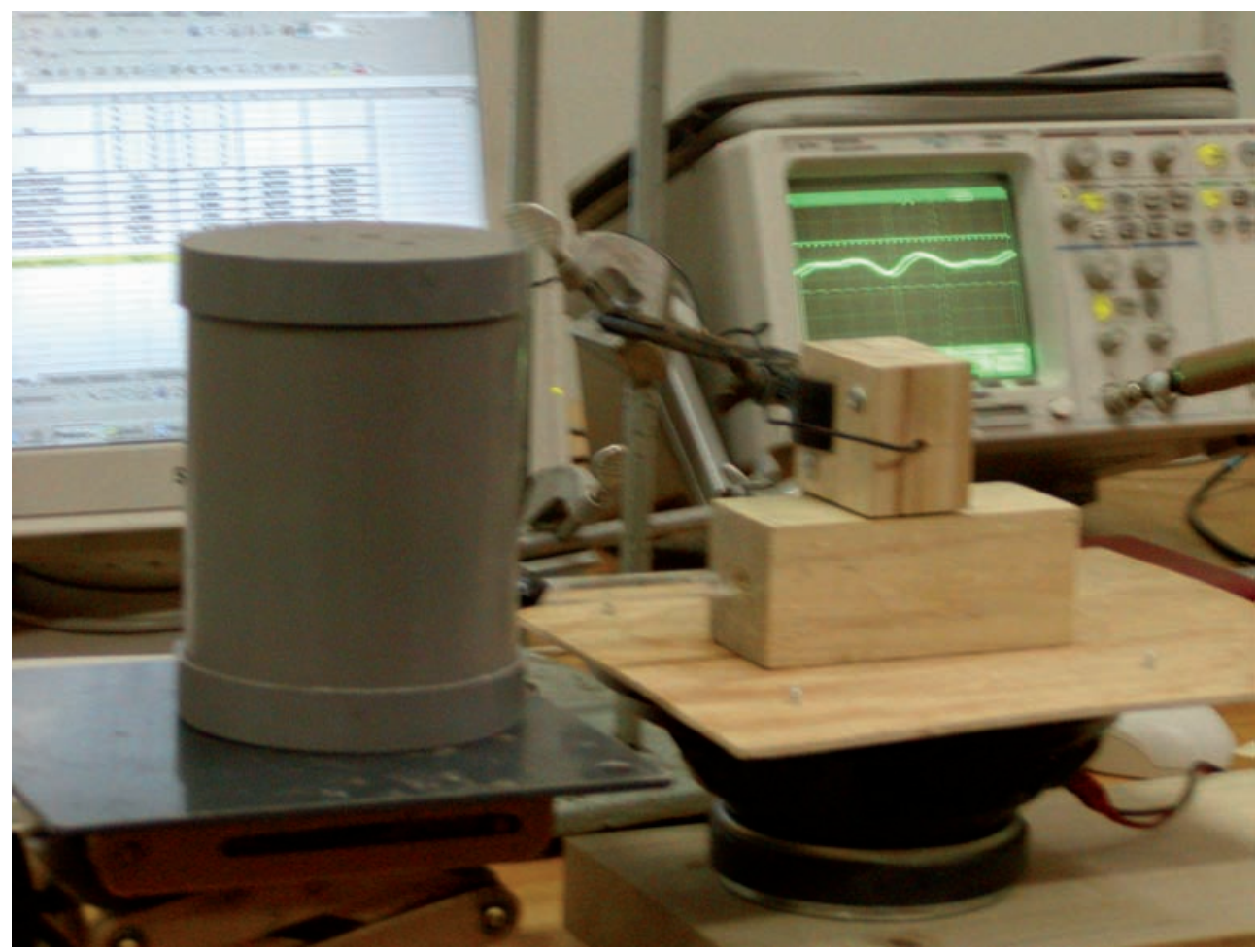

Figura 2. Set experimental de ensayos sónicos. 
Ensayos previos mostraron que la frecuencia de resonancia de las termitas fue de $80 \mathrm{~Hz}$, adoptándose ésta frecuencia para hacer vibrar probetas en escuadrías de $19 \times 41 \mathrm{~mm}, 41 \mathrm{x} 41 \mathrm{~mm}$ y $41 \mathrm{x} 90 \mathrm{~mm}$ de una longitud de $10 \mathrm{~cm}$ en cortes tangencial, radial y mixto y en tres niveles de densidad (baja, media y alta).

Una vez establecido un flujo constante de termitas se procedió a excitar las probetas utilizando un parlante de $80 \mathrm{hm}$ y una potencia de $50 \mathrm{~W}$, en el cual se instaló una madera contrachapada de $3 \mathrm{~mm}$ de espesor como membrana, que se hizo vibrar a $80 \mathrm{kHz}$, variando la intensidad de modo de mantener una amplitud constante de $3 \mathrm{~V}$.

Los equipos utilizados fueron un generador de onda de $20 \mathrm{MHz}$, modelo $33220 \mathrm{~A}$, un osciloscopio digital de doble canal de $100 \mathrm{MHz}$, un micrófono de condensador y un PC con los programas apropiados para el análisis de señales. Como fuente de potencia se utilizó un amplificador CS-1200 X de 600W por canal.

A objeto de controlar la acumulación de termitas en el interior de la muestra y verificar el efecto de los tratamientos en la actividad de las termitas se midieron cuatro flujos de tránsito, dos desde el nido hacia la fuente (a la entrada y salida de la probeta) y dos flujos desde ésta última hasta el nido, determinándose en un tiempo total de 15 minutos durante un período de dos horas, con repetición de las mediciones durante tres días consecutivos. En el set que se mantuvo de testigo, las mediciones de flujo se efectuaron en forma simultánea correspondientes con los periodos de excitación.

Para efectuar el conteo de termitas durante 15 minutos de tránsito, se filmó mediante una cámara de video de modo de facilitar las mediciones al proyectar el sistema a una escala mayor.

\section{RESULTADOS Y DISCUSIÓN}

Ensavos Ultrasónicos: Los resultados de estos ensayos permitieron cuantificar el porcentaje de sobrevivencia y evaluar el efecto del ultrasonido en la capacidad de alimentación de las termitas. En la tabla 2 se presentan los porcentajes de supervivencia promedio para las distintas frecuencias utilizadas y para las probetas testigos.

La Norma EN 118 fija como válidos los ensayos en que dos de tres testigos alcanzan una supervivencia mayor a un $50 \%$ de los individuos. Lo que en este ensayo fue logrado, puesto que en las termitas testigos se obtuvo una supervivencia promedio de $54 \%$.

Tabla 2 Supervivencia promedio de las termitas para cada frecuencia.

\begin{tabular}{|c|c|c|}
\cline { 2 - 3 } \multicolumn{1}{c|}{} & \multicolumn{2}{c|}{ Supervivencia } \\
\hline Tratamientos $[\mathbf{k H z}]$ & $\mathbf{N}^{\circ}$ de Individuos & $\%$ \\
\hline 100 & 142 & 56,8 \\
\hline 500 & 154 & 61,6 \\
\hline 1000 & 86 & 34,4 \\
\hline 0 (Testigos) & 135 & 54,0 \\
\hline
\end{tabular}

Para la frecuencia de $100 \mathrm{kHz}$ y $500 \mathrm{kHz}$ la supervivencia fue muy similar y superior al $50 \%$, a diferencia de la frecuencia de $1000 \mathrm{kHz}$ en que se obtuvo la menor sobrevivencia, $34 \%$, sin embargo, el ataque a la madera correspondió a un ataque fuerte como se puede apreciar en el Tabla 3 donde se presentan los grados de ataque promedio sufrido por las probetas según Norma EN 118. Cabe hacer notar que las probetas ensayadas con frecuencia de $500 \mathrm{kHz}$ sufrieron un ataque clasificado como medio grado 3 , que corresponde a un deterioro superficial que se extiende a más de un cuarto de la superficie expuesta, no obstante, es el tratamiento que presenta el mayor porcentaje de supervivencia, $61,6 \%$. 
Tabla 3. Grado de ataque promedio sufrido por las probetas según Norma EN 118.

\begin{tabular}{|c|c|}
\hline Probeta & Grado de ataque $^{*}$ \\
\hline $100 \mathrm{kHz}$ & 4 \\
\hline $500 \mathrm{kHz}$ & 3 \\
\hline $1000 \mathrm{kHz}$ & 4 \\
\hline Testigos & 4 \\
\hline
\end{tabular}

* 3 = medio; 4 = fuerte

Antes de entrar a analizar esta aparente contradicción, es necesario destacar algunas particularidades observadas en el curso de los experimentos. Como ya se estableció los tubos de ensayos fueron llenados con sustrato hasta 7 centímetros de altura, la que se incrementó en dos centímetros en las probetas testigos y en un centímetro en las probetas tratadas; esto fue debido al desplazamiento del sustrato, producto de la actividad de las termitas, en el proceso de construcción de galerías a través de él con el objeto de acceder a la probeta para alimentarse. Esto indica que existió un efecto del ultrasonido sobre las partículas del sustrato, productos de las vibraciones produciéndose una mayor compactación de este. Por otra parte se observó que transcurrido una semana de los ensayos apareció la formación de galerías en las paredes de los tubos de las probetas tratadas, lo que no sucedió con los testigos, lo que podría llevar a considerar que la presencia del campo acústico ocasionó un efecto molestia que incentivó la tendencia de geotropismo negativo de las termitas. Otro efecto importante que se evidenció fue que mientras se mantuvo el campo ultrasónico las termitas suspendieron su alimentación subiendo por el sustrato hasta una altura mínima de $50 \mathrm{~mm}$ sobre la probeta, reanudándose el ataque una vez que la irradiación se detuvo, lo que mostró claramente el efecto de barrera que produjo el ultrasonido.

En relación al efecto de las frecuencias en el porcentaje de supervivencia y grado de ataque a la madera, se concluye que en el caso de la frecuencia de $100 \mathrm{kHz}$, ésta no provocó un efecto significativo sobre las termitas durante el tiempo que ellas fueron irradiadas. En la frecuencia de $500 \mathrm{kHz}$, donde existió una mayor supervivencia y un menor ataque, se postula que ello se debió a que las termitas fueron más afectadas en la facilidad al acceso al alimento, o bien existió alguna influencia en el proceso metabólico el que habría influido en la tasa de alimentación, sin que ello produjera efectos letales; cabe al respecto considerar el hecho que la alteración metabólica se haya producido por la absorción del ultrasonido por el ácido acético que participa en el proceso de digestión. Para una frecuencia de $1000 \mathrm{kHz}$, en que se obtuvo una menor supervivencia pero un ataque severo, las termitas en este caso se deben haber mantenido muy cercanas a la interfase sustrato-madera durante la irradiación, en mayor atenuación que se produce a estas altas frecuencias, no habiéndoles producido mayores alteraciones, sin embargo, ello habría significado que ellas fueran sometidas a una mayor irradiación lo que le habría producido efectos letales por un mayor tiempo acumulado de expansión. Cabe hacer notar que el efecto de ultrasonido en los tejidos biológicos no solo depende de la intensidad de irradiación y de la frecuencia sino que también del tiempo de exposición (Kenneth, 1988) 
Irradiación en Agua: Los resultados obtenidos con la metodología propuesta mostraron que a partir de los 10 segundos de irradiación ya se observaron para las dos frecuencias y las tres energías consideradas, destrucción de algunas termitas, incrementándose en forma paulatina la mortalidad con el tiempo de irradiación (durante el cual no existió incremento de temperatura del agua), lo que conduce a afirmar que en todos los tratamientos se produjo el fenómeno de cavitación siendo ésta la causa directa de la muerte de las termitas, con una destrucción brutal de sus tejidos, sin embargo, no fue posible establecer si la cavitación se produjo en el medio acuoso o en los intersticios intercelulares de los insectos. Respecto al efecto del agua en las termitas se comprobó que no tuvo ninguna influencia directa sobre ellas en ausencia de irradiación, observándose en los testigos una supervivencia de $100 \%$ después de haber estado sumergidas en agua destilada a $20^{\circ} \mathrm{C}$, durante los tiempos de tratamiento, recuperando su actividad normal después de aproximadamente 15 minutos de ser retiradas de los depósitos.

En el Tabla 4 es posible apreciar que la mortalidad promedio de las termitas para cada tratamiento aplicado tuvo como resultado un alto grado de mortalidad, llegando en promedio al 91\%. Así mismo es posible observar que en todos los ensayos realizados existen tratamientos que conducen a obtener un $100 \%$ de mortalidad.

Tabla 4. Mortalidad de las termitas (\%) para las distintas frecuencias, potencias y tiempos de aplicación.

\begin{tabular}{|c|c|c|c|c|c|c|c|c|c|}
\hline \multirow{4}{*}{ FRECUENCIA (A) } & \multicolumn{9}{|c|}{ POTENCIAS (B) } \\
\hline & \multicolumn{3}{|c|}{$5 W$} & \multicolumn{3}{|c|}{$10 \mathrm{~W}$} & \multicolumn{3}{|c|}{$15 \mathrm{~W}$} \\
\hline & \multicolumn{9}{|c|}{ TIEMPOS (C) } \\
\hline & $2 \min$ & $5 \mathrm{~min}$ & $10 \mathrm{~min}$ & $2 \mathrm{~min}$ & $5 \mathrm{~min}$ & $10 \mathrm{~min}$ & $2 \min$ & $5 \min$ & $10 \mathrm{~min}$ \\
\hline $11 \mathrm{kHz}$ & 93 & 87 & 100 & 90 & 97 & 100 & 100 & 100 & 93 \\
\hline $20 \mathrm{kHz}$ & 33 & 50 & 100 & 90 & 100 & 100 & 100 & 100 & 100 \\
\hline
\end{tabular}

En relación a la mortalidad de las termitas la prueba de intervalos de Duncan para la potencia determinó que existen diferencias significativas entre la potencia de $15 \mathrm{~W}$ con respecto a la de $10 \mathrm{~W}$ y $5 \mathrm{~W}$. Así mismo se determinó que existen diferencias significativas de los 10 minutos de exposición. Entre los tiempos de cinco minutos y dos minutos no existieron diferencias estadísticamente significativas.

Del análisis de varianza a un nivel de confianza del 95\% que se muestra en el Tabla 5 se obtuvo que la mortalidad de las termitas depende de la frecuencia, de la potencia de irradiación aplicadas y del tiempo de exposición. 
Tabla 5. Análisis de varianza para mortalidad de los individuos.

\begin{tabular}{|c|c|c|c|c|}
\hline Fuente de Variación & Suma de Cuadrados & $\begin{array}{c}\text { Grados de } \\
\text { Libertad }\end{array}$ & $\begin{array}{c}\text { Media de } \\
\text { Cuadrados }\end{array}$ & Fo \\
\hline Frecuencia (A) & 12,52 & 1 & 12,52 & $11,46^{*}$ \\
\hline Potencia (B) & 50,04 & 2 & 25,02 & $22,90^{*}$ \\
\hline Tiempos (C) & 19,70 & 2 & 9,85 & $9,02^{*}$ \\
\hline AB & 34,48 & 2 & 17,24 & $15,78^{*}$ \\
\hline$A C$ & 11,26 & 2 & 5,63 & $5,15^{*}$ \\
\hline $\mathrm{BC}$ & 31,63 & 4 & 7,91 & $7,24^{*}$ \\
\hline ABC & 16,74 & 4 & 4,19 & $3,83^{*}$ \\
\hline Error & 39,33 & 36 & 1,09 & \\
\hline Total & 215,70 & 53 & & \\
\hline
\end{tabular}

Debe hacerse notar que el experimento desarrollado tuvo la desventaja de utilizar un transductor plano para producir el campo acústico, ya que la interfase líquido-sólido constituye un sitio preferencial para la formación de burbujas, dando como resultado final que se genere muy rápidamente cavitación acústica sobre la cara del transductor disminuyendo la intensidad del sonido del sistema, debido a que se produce una alta atenuación al pasar por ésta región y en consecuencia la sonificación es altamente reducida en la masa del líquido. Este efecto puede ser reducido enfocando el campo mediante lentes o bien utilizando transductores de superficie cóncava (Kenneth, 1988).

Ensayos de Barrera Sónica: Una vez establecido un flujo de termitas relativamente estable a través de las muestras de madera instaladas en el mini termitero, lo que se logró en un período de 15 días una vez establecido, se procedió a efectuar las observaciones tanto en el set experimental como en el testigo, en forma simultánea.

Se hace notar que se entregan aquí solo los resultados cualitativos obtenidos, en razón a que los ensayos se encuentran en desarrollo lo que no permite aún obtener resultados que sean concluyentes.

Los resultados obtenidos, de carácter parcial, mostraron que con una frecuencia de oscilación de $80 \mathrm{~Hz}$ se produjo, en todas las muestras consideradas, una notoria alteración del flujo de termitas mientras la vibración se encontraba presente, deteniéndose prácticamente en su totalidad, después de 15 minutos, el paso de las termitas en ambos sentidos (desde el nido a la muestra y desde la fuente de alimentación hacia la muestra), manteniéndose ésta situación durante todo el período de excitación.

Después de un tiempo acumulado de dos horas de tratamiento, (cuatro tratamientos de treinta minutos con intervalos de una hora) y después de una hora finalizado el último tratamiento el nivel de actividad de las termitas disminuyó drásticamente en relación al testigo y a las condiciones iniciales antes del primer tratamiento, siendo ello cada vez más notorio en los dos días consecutivos en que se repitió el ensayo.

Después de transcurrida una semana la actividad observada fue normal, con una ligera disminución del número de termitas por minuto que pasaban a través de la probeta.

A éste nivel de avance de los ensayos no fue posible establecer un efecto sobre la capacidad de alimentación de las termitas y la mortalidad causada por las vibraciones. 


\section{CONCLUSIONES}

Los ensayos ultrasónicos mostraron claras evidencias que la irradiación a las tres frecuencias aplicadas constituyeron una barrera al acceso de las termitas a su fuente de alimentación.

La irradiación en agua provocó la mortalidad de las termitas por efecto de cavitación para las frecuencias e intensidades de energía consideradas.

Las vibraciones mecánicas a una frecuencia de $80 \mathrm{~Hz}$, permiten establecer una barrera física al paso de las termitas mientras la excitación se encuentra presente.

\section{AGRADECIMIENTOS}

Los autores agradecen a FONDECYT por el financiamiento del proyecto $\mathrm{N}^{\circ} 1040726$ en cuyo contexto se realizó este estudio.

\section{BIBLIOGRAFÍA}

Almagro, V. 1967. Los ultrasonidos. Editorial Paraninfo. Madrid España.

Camousseight, A. 1998. Las termitas y su presencia en Chile, Folleto de divulgación CONAF.

Child, S.Z.; Christensen, E.L.; Smarchlo, K. 1989. Effects of ultrasound on Drosophilae. Killing of eggs exposed to traveling and standing wave fields. Ultrasound Med. Biog. 6: $127-130$.

De Groot, R.C.; Ross, R. J.; Nelson, W.T. 1998. Non-Destructive Assessment of Wood Decay and termite attack in Southern pine sapwood. Wood Protection 3:25-34.

Delate, K.M.; Grace, F.K; Armstrong, J.W.1995. Carbon dioxide as a potential fumigant for termite control. Pesticide Science 44: 357-361.

Grace, J.K.; Wood, D.L.; Kubo, I.; Kim, M. 1995. Behavior and Chemical investigation of trail pheromone from the termite Reticulitermes hesperus Banks (Isoptera Rhinotermitidae). Journal of applied Entomology 199: 501-505

Hungate, R.E; 1975. La celulosa en la nutrición animal. Compañía editorial continental. Primera edición en español 36 p.

Instituto de Investigación Agropecuarias (INIA). 2004. Termitas y otros insectos xilófagos en Chile: Especies, Biología y Manejo. Colección Libros INIA No 11. Editores. Renato Ripa y Paola Luppichini.

Intec-Chile-Infor. 1997. Un nuevo desafio al uso intensivo de la madera: La termita subterránea. Estudio de su impacto y prevención. Proyecto FDI- CORFO 96C1-FP24.

Kenneth, S.S.1988. Ultrasound. Its Chemical, Physical, and biological effects. Edited by Kenneth S. Suslick. VCH. Publishers. North America.

Lemaster, R.I.; Beall, F.C.; Lewis V.R. 1997. Detection of termites with acoustic emission. For. Prod. J. 47:75-79 
Lewis, V.R. 1997. Alternative control strategies for termites. Journal of Agricultural Entomology 14:291- 307.

Mankin, R.W.; Osbrink, W.L.; Oi, F.M.; Anderson, J.B. 2002. Acoustic detection of termite infestations in urban trees. J. Ecom. Entomol. 95(5):981-988.

Mashek, B. 1998. Drywood termite control. Microwaves at work I.P.M. ww.nrthwesttermite.com/ appteen.html.

Morris, P.I. 2000. Integrated control of subterranean termites: The 6s approach group leader- Durability and Protection group. Forintek, Canada corporation, Vancouver, B.C. VGTIWO

Norma Española UNE. 2005. Protectores de la madera. Determinación de la eficacia preventiva contra las especies de reticulitermes (termitas europeas). Método de laboratorio. UNE- EN118:2005

Ross, R.J.; Degroot, R.C.; Nelson, W.J.; Le Bow, P.K. 1997. The relationship between stress wave transmission characteristics and the compressive strength of biologically degraded woods. Forest Prod. J. 47(5):89-93.

Shutilov, V.A. 1988. Fundamental physics of ultrasound. Gordon and Bregan Science Publishers.S.A. Great Britain

Smith, JL.; Rust, M.K. 1993a. Influence of temperature on tunneling, feeding rates, and oxygen requirement of the western subterranean termite, Reticulitermes hesperus (Isoptera:Rhinotermitidae). Sociobiology 21(3):225-235.

Smith, JL.; Rust, M.K. 1993b. Effect of relative humidity and temperature on the survival of Reticulitermes hesperus (Isoptera, Rhinotermitidae) Sociobiology 21:217-224.

Smith, JL.; Rust, M.K. 1994. Temperature preference of the western subterranean termite. Reticulitermes hesperus Banks. Journal of arid environments 28:313-323.

Wilcox, W.W 1988. Detection of early stages of wood. Decay with ultrasonic pulse velocity. Forest Prod. J. 38(5): 68-73

Woodrow, R.J.; Grace, J K. 1988. Thermal tolerances of four termite species (Isoptera: Rhinotermitidae, Kalotermitidae) Sociobiology 32:17-25.

Yanase, Y.; Fuji, Y.; Okumura, Y.; Imamura, T.; Yoshimura,T. 1998. Detection of AE generated by the feeding activity of termites using PDVF. Forest Prod. J. 48(7-8): 43-46. 
